Rev. Biol. Trop., 46(3):525-532, 1998

\title{
Estructura y sucesión en bosques montanos del Noroeste de Argentina
}

M.F. ${ }^{1,2}$ Arturi, H.R. Grau ${ }^{1,3}$, P. G. Aceñolaza ${ }^{1}$ y A.D. Brown ${ }^{1}$

' Laboratorio de Investigaciones Ecológicas de las Yungas, Universidad Nacional de Tucumán. Casilla de Correo 34, (4107) Yerba Buena, Tucumán, Argentina. Fax: 54-81-304957. E-mail: liey@untlie.edu.ar

2 Dirección actual: Laboratorio de Investigación en Sistemas Ecológicos y Ambientales, Facultad de Ciencias Agraria y Forestales, Universidad Nacional de La Plata. Diag. 113 No 469, (1900), La Plata, Buenos Aires, Argentina. E-mail: lisea@isis.unlp.edu.ar

Dept. of Geography. Campus Box 260. University of Colorado, 80309, Boulder, Colorado, USA. Fax: (303)492-7501. Email: grau@ucsu.colorado.edu

Recibido 21-V-1997. Corregido 27-V-1998. Aceptado 19-VI-1998.

\begin{abstract}
Floristic composition and tree size distribution of different forest patches were studied between 1600 and $1800 \mathrm{~m}$ altitude in the Subtropical Montane Forests of Parque Biológico Sierra de San Javier, Argentina. Quadrats (20 x $20 \mathrm{~m}$ ) were sampled in 12 sites. Sites were ordered using correspondence analysis. The first ordination axis was clearly associated to a gradient in complexity and diversity. The less complex forests were dominated by Alnus acuminata, Podocarpus parlatorei, and Crinodendron tucumanum. Richest and most complex forests were dominated by Myrtaceae species such as Blepharocalyx salicifolius, Myrcianthes mato and Myrcianthes pseudomato, accompanied by Cedrela lilloi, Ilex argentina, Prunus tucumanensis, and others. Intermediate values of diversity and complexity were found in mature forests of A. acuminata and P. parlatorei, and in forests dominated by the treelets Dunalia lorentzii, Sambucus peruviana and Solanum grossum. Sites were grouped using the Twinspan software package. Within each cluster, diameter distribution of the most important species was used to describe regeneration trends. Myrtaceae species showed an "inverted-J" distribution of diameter, suggesting a continous regeneration mode. On the other hand, species which dominate simple forests such as $A$. acuminata, P. parlatorei, and C. tucumanum showed bell-shaped diameter distribution, suggesting lack of regeneration under the canopy. These conclusions were also supported by seedling and sapling densities. The results suggest that $A$. acuminata, P. parlatorei, and C. tucumanum are pioneer species, and that the forests dominated by such species were originated in grasslands or shrublands. These species seems to be gradually replaced by shade tolerant taxa originating patchy heterogeneity and landcape diversity as a result of discrete events of recruitment followed by forest succession.
\end{abstract}

Key words: Montane forests, cloud forests, forest structure, succesion, disturbance, Yungas, Argentina.

Los Bosques Montanos del Noroeste argentino, constituyen un distrito dentro de la Provincia Fitogeográfica de Las Yungas (Cabrera 1976). En Argentina, estos bosques se extienden desde el límite con Bolivia a $22^{\circ} \mathrm{S}$ hasta aproximadamente $29^{\circ} \mathrm{S}$ en la Provincia de Catamarca y desde los 1600 a más de 2500 msnm. A escala de paisaje, esta unidad biogeográfica es un mosaico de diferentes unidades de vegetación entre los que se destacan los pastizales, los arbustales 
dominados por compuestas y los bosques simples de Alnus acuminata, Podocarpus parlatorei, Sambucus peruviana y Polylepis australis. Hacia el límite altitudinal inferior los bosques son más diversos formados principalmente por P. parlatorei, Ilex argentina, Prunus tucumanensis, Cedrela lilloi, Juglans australis y diversas mirtáceas (Cabrera 1976, Hueck 1978). Estas fisonomías han sido descritas como comunidades estables. Sin embargo, Grau (1985) ha documentado que en numerosas áreas el pastizal ha sido reemplazado por bosques de Alnus acuminata en las últimas décadas. Los parches de distintos tipos de bosque podrían mantenerse vinculados a un régimen de disturbios y sucesión como ocurre en otros sistemas (Pickett y. White 1985). Las relaciones dinámicas entre los distintos tipos de bosque no han sido aún estudiadas. El conocimiento de la dinámica de parches en relación con disturbios naturales y antrópicos es importante para el diseño y manejo de áreas protegidas (Romme 1982, Baker 1992, Turner et al. 1994). Para ello es necesario entender las propiedades temporales y espaciales del régimen de disturbios y los procesos sucesionales subsiguientes. Los Bosques Montanos de las Yungas Argentinas constituyen un área fuertemente afectada por disturbios antrópicos (Grau 1989, Molinillo y Vides-Almonacid 1989) y tienen una escasa superficie incluida en reservas (Brown 1995).

En este trabajo se describe la estructura de distintas fisonomías del Bosque Montano. Sobre la base de estos datos se evalúa la hipótesis de que parches con distinta fisonomía y composición pueden asimilarse a una cronosecuencia sucesional y que diferentes composiciones reflejan el reemplazo sucesional de especies pioneras por especies tolerantes a la sombra (sensu Swaine y Whitmore 1988). Bajo este modelo, los parches dominados por especies pioneras, se mantendrían mediante un modo de regeneración "catastrófico" y progresivamente serían reemplazados por especies con modos de regeneración en fase de "claro" o "continua" (sensu Veblen 1992).

\section{MATERIAL Y METODOS}

El estudio se llevó a cabo en los Bosques Montanos del Parque Biológico Sierra de San Javier, área reservada de la Universidad Nacional de Tucumán, $27^{\circ} \mathrm{S}$, en la provincia de Tucumán, Argentina. El clima del área se caracteriza por una temperatura anual de 14 a $15^{\circ} \mathrm{C}$ y 1 400-1 600 mm de precipitación. Biogeográficamente, la zona se considera parte del distrito de los bosques montanos, de la provincia fitogeográfica de las Yungas (Cabrera 1976). Los muestreos se realizaron entre los 1600 y 1800 msnm en la zona de cumbre de la Sierra de San Javier, que es un mosaico de unidades fisonómicas de bosque alternadas con pastizal (Moyano y Movia 1989).

Se seleccionaron 12 sitios correspondientes a distintas fisonomías de este piso altitudinal mediante fotografías aéreas y y reconocimiento en el terreno. Se utilizaron parcelas contiguas de $20 \times 20 \mathrm{~m}$ en un número que varió entre 4 y 12 por sitio (Cuadro 1). La superficie total muestreada, incluyendo todos los sitios, fue de 3.24 ha. Cada individuo muestreado fue determinado a nivel de especie e identificado con una marca metálica para su seguimiento en parcelas permanentes. Se registró el diámetro del tallo a la altura del pecho (DAP) de todos los árboles de 10 o más $\mathrm{cm}$ de DAP. La altura de cada árbol se estimó visualmente. Se calculó la densidad (ind/ha), el área basal ( $\mathrm{m}^{2} / \mathrm{ha}$ ), la frecuencia y el indice de valor de importancia (IVI = densidad relativa [\%] + área basal relativa [\%] + frecuencia relativa [\%]) para cada especie registrada. Para cada sitio se calculó el índice de altura (media de los tres árboles más altos) y el índice de complejidad (Holdridge 1978).Los individuos menores de $10 \mathrm{~cm}$ de DAP fueron muestreados distinguiendo dos clases de tamaño: renovales menores (individuos menores de $3 \mathrm{~cm}$ de DAP y mayores de $50 \mathrm{~cm}$ de altura) y renovales mayores (individuos de entre 3 y $10 \mathrm{~cm}$ de DAP). En cada sitio se utilizaron 30 parcelas de $4 \times 5 \mathrm{~m}$ para los renovales menores y cinco parcelas de $20 \times 20 \mathrm{~m}$ para los mayores.

Los distintos sitios fueron ordenados mediante análisis de correspondencia (ter Braak 
1987) utilizando valores de IVI para cada especie calculado con los individuos mayores de 10 $\mathrm{cm}$ de DAP. Al mismo juego de datos se le aplicó un método divisivo de clasificación (Twinspan, Hill 1979). Este método se aplicó con la finalidad de agrupar sitios con similar composición específica. Este mismo análisis se realizó con los valores de densidad de los renovales mayores y menores.

Se estudió la distribución de diámetros de las especies más abundantes en cada grupo de sitios formado por Twinspan con la finalidad de inferir tendencias en la dinámica poblacional.

\section{RESULTADOS}

Los sitios muestreados presentaron un gradiente de riqueza y complejidad desde los bosques simples dominados por Alnus acuminata, hasta los bosques más complejos dominados por mirtáceas como Myrcianthes mato, Myrcianthes pseudomato y Blepharocalyx salicifolius (Cuadro 1). Los dos primeros ejes del análisis de correspondencia acumularon el $55 \%$ de la varianza. La distribución de los sitios, y los grupos de sitios definidos por Twinspan, a lo largo del primer eje se asoció con el gradiente de complejidad y riqueza. Los bosques de menor riqueza y complejidad, dominados por $A$. acuminata (grupo A)(Cuadro 2), se ubicaron en el extremo positivo (Fig. 1). En una posición central se agruparon los bosques de riqueza y complejidad intermedias caracterizados por $P o$ docarpus parlatorei y Crinodendron tucumanum y sitios maduros de A. acuminata (grupo B). En el grupo $C$ se presentaron sitios con gran importancia de especies de árboles del estrato arbóreo inferior como Sambucus peruviana, Dunalia lorentzii y Solanum grossum. Los bosques más complejos y diversos, con especies como B. salicifolius, M. mato, Juglans australis, Cedrela lilloi y Allophylus edulis (grupo D), se ubicaron en el extremo negativo del primer eje. Las especies más asociadas con el primer eje fueron A. acuminata, B. salicifolius y $M$. mato.
La distribución de los sitios en el segundo eje de ordenamiento respondió principalmente a las abundancias de las especies del estrato arbóreo inferior $(D$. lorentzii, S. peruviana y $S$. grossum). Los sitios con mayor proporción de estas especies (grupo C) aparecieron en el extremo negativo.
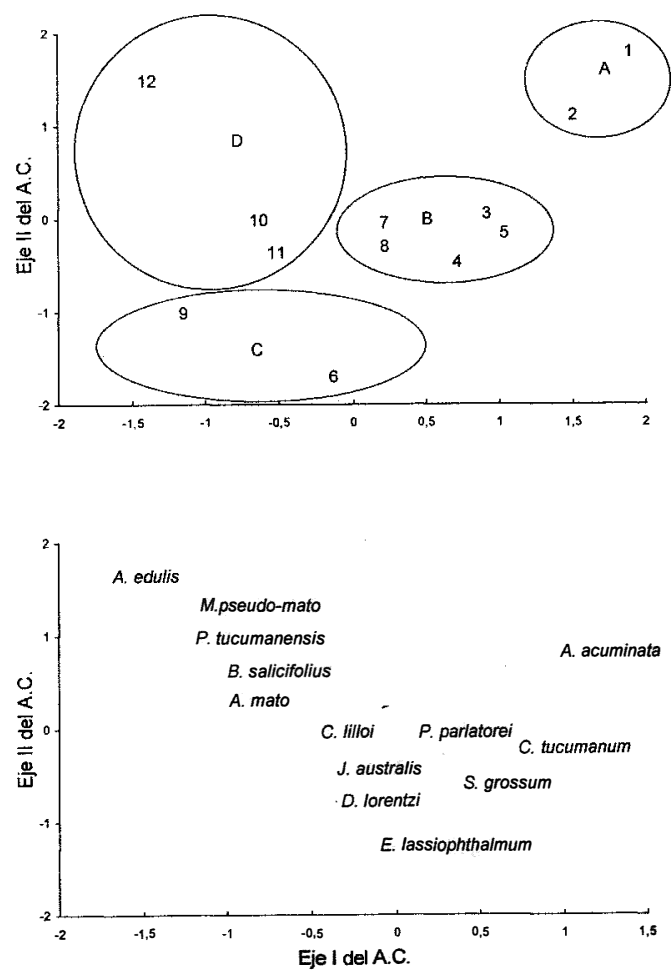

Fig. 1. Distribución de los sitios y especies en los dos primeros ejes del análisis de correspondencia. A, B, C y D grupos obtenidos por análisis de clasificación (Twinspan). Sites and species distribution for two first axes of correspondence analysis. A, B, C and D: classification groups (Twinspan). 


\section{CUADRO 1}

Superficie muestreada (ha) y variables estructurales para cada sitio en los Bosques Montanos del NO de Argentina.. Sampled area (ha) and structural variables for each site in montane forests, NW Argentina.

\begin{tabular}{|c|c|c|c|c|c|c|c|}
\hline Sitios & ha & D & $\mathrm{AB}$ & $\mathrm{h}$ & $S p$ & IC & IC Medio \\
\hline 1 & 0.24 & 500 & 15.7 & 9 & 4 & 2.8 & A: 39.3 \\
\hline 2 & 0.24 & 322 & 22.4 & 14 & 5 & 5.0 & B: 90.4 \\
\hline 3 & 0.24 & 326 & 29.3 & 18 & 6 & 10.3 & C: 14.4 \\
\hline 4 & 0.24 & 267 & 20.2 & 10 & 4 & 2.2 & D: 33.7 \\
\hline 5 & 0.24 & 214 & 21.3 & 10 & 4 & 1.9 & \\
\hline 7 & 0.16 & 425 & 32.8 & 14 & 3 & 5.9 & \\
\hline 8 & 0.24 & 498 & 42.1 & 17 & 7 & 25.0 & ' \\
\hline 6 & 0.24 & 662 & 27.2 & 15 & 6 & 16.2 & \\
\hline 9 & 0.24 & 300 & 30.0 & 20 & 7 & 12.6 & \\
\hline 10 & 0.24 & 400 & 53.6 & 19 & 10 & 40.7 & \\
\hline 11 & 0.48 & 391 & 35.0 & 17 & 10 & 23.3 & \\
\hline 12 & 0.40 & 410 & 39.0 & 18 & 13 & 37.4 & \\
\hline
\end{tabular}

D: densidad (ind/ha), AB: área basal ( $\left.\mathrm{m}^{2} / \mathrm{ha}\right)$, h: índice de altura $(\mathrm{m}), \mathrm{Sp}$ : número de especies, IC: índice de complejidad, IC Medio: índice de complejidad promedio para todos los sitios incluidos en los grupos de clasificación (A, B, C y D).

D: density (tree/ha), AB: basal area ( $\left.\mathrm{m}^{2} / \mathrm{ha}\right)$, h: height index $(\mathrm{m}), \mathrm{Sp}$ : species richness, IC: complexity index, IC Medio: mean complexity index for all sites within each group (A, B, C and D)

\section{CUADRO 2}

Indice de Valor de Importancia para cada especie en cada sitio en los Bosques Montanos del NO de Argentina. Importance value index per species for each site in montane forests, NW Argentina.

Especies

\section{Alnus acuminata H.B.K}

Podocarpus parlatorei Pilg.

Crinodendron tucumanum Lillo

Sambucus peruviana H.B.K.

Dunalia lorentzii (Daumner) Sleumer

Solanum grossum Morton

Eupatorium lasiophthalmum Griseb.

Juglans australis Griseb.

Cedrela lilloi C.DC

Blepharocalyx salicifolius (H.B.K)

O.Berg.

Myrciantes mato Griseb Mc Vaugh

Myrciantes pseudomato (Legrand) Mc Vaugh

Myrciantes callicoma Mc Vaugh

Duranta serratifolia (Griseb.) Kuntse

Prunus tucumanensis Lillo

Ilex argentina Lillo

Allophylus edulis (St. Hill) Radlk

Myrsine laetevirens (Mez) Arechav.

Schinus gracilipes Johnst.

Celtis iguanea (Jacq.) Sarg.

Azara salicifolia Griseb.
Vassobia breviflora (Sendnt.) Hunz.

Sitios

$\begin{array}{rrrrrr}1 & 2 & 3 & 4 & 5 & 6 \\ 290 & 246 & 164 & 49 & 122 & 0 \\ 40 & 89 & 95 & 123 & 0 & 57 \\ 0 & 59 & 47 & 210 & 195 & 0 \\ 18 & 56 & 140 & 0 & 95 & 180 \\ 0 & 0 & 84 & 0 & 0 & 158 \\ 19 & 100 & 136 & 117 & 105 & 118 \\ 0 & 0 & 0 & 0 & 0 & 54 \\ 0 & 0 & 0 & 0 & 0 & 0 \\ 0 & 0 & 0 & 0 & 0 & 0\end{array}$

Las letras indican el grupo al que pertenece cada especie según la densidad de los renovales menores (Me) y mayores (Ma). *: especie sólo presente como renovales menores, ** especie sólo presente como renoval mayores, nc: especie no clasificada debido a su baja frecuencia.

Letters indicate the classification group based on seedlings (Me) and saplings (Ma) density for each species. *: species only present as seedlings,

**: species only present as saplings, nc: species not classified due to its low frequency.
Men May

$\begin{array}{rrrrrrrr}7 & 8 & 9 & 10 & 11 & 12 & & \\ 27 & 19 & 0 & 0 & 0 & 0 & \text { A } & \text { A } \\ 291 & 277 & 164 & 248 & 43 & 119 & \text { A } & \text { A } \\ 0 & 81 & 115 & 0 & 125 & 16 & \text { A } & \text { A } \\ 57 & 36 & 127 & 74 & 97 & 76 & \text { B } & \text { B } \\ 0 & 0 & 92 & 18 & 72 & 47 & \text { C } & \text { A } \\ 0 & 18 & 119 & 38 & 117 & 0 & \text { B } & \text { B } \\ 0 & 0 & 0 & 0 & 9 & 0 & \text { B } & \text { B } \\ 0 & 18 & 79 & 78 & 41 & 0 & \text { D } & \text { C } \\ 0 & 18 & 37 & 0 & 0 & 22 & \text { D } & \text { C }\end{array}$

$\begin{array}{llllllllllllll}0 & 0 & 0 & 0 & 0 & 0 & 0 & 0 & 0 & 55 & 41 & 70 & \mathrm{C} & \mathrm{C}\end{array}$

$\begin{array}{llllllllllllll}0 & 0 & 0 & 0 & 0 & 0 & 0 & 0 & 0 & 84 & 134 & 137 & \mathrm{C} & \text { nc }\end{array}$

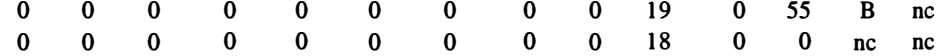

$\begin{array}{llllllllllllll}0 & 0 & 0 & 0 & 0 & 0 & 0 & 0 & 0 & 0 & 0 & 84 & \text { D } & \text { C }\end{array}$

$\begin{array}{llllllllllllll}0 & 0 & 0 & 0 & 0 & 0 & 0 & 0 & 0 & 0 & 39 & 55 & \text { B } & \text { nc }\end{array}$

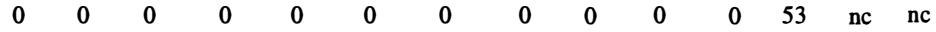

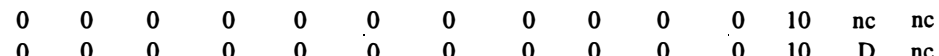

0

*

nc

nc

B 
En el grupo A, la especie dominante, $A$. acuminata, y las principales acompañantes $(P$. parlatorei y $C$. tucumanum) presentaron la mayor densidad de individuos en las clases diamétricas menores a $20 \mathrm{~cm}$ (Fig. 2 A). En el grupo B, estas especies también mostraron una distribución unimodal de diámetros, aunque con mayor proporción de inviduos mayores de $20 \mathrm{~cm}$ de DAP (Fig. 2 B). En el grupo C, C. tucumanum y $P$. parlatorei presentaron distribuciones diamétricas menos definidas pero con mayores diámetros que en el grupo B. A. acuminata estuvo ausente en los grupos $\mathrm{C}$ y $\mathrm{D}$.

En el-grupo D se presentaron las especies $B$. salicifolius y $M$. mato con máximos de densidad en las clases menores de $20 \mathrm{~cm}$ de DAP respectivamente, aproximándose a una distribución diamética en "J invertida" (Fig. 2 D). C. tucumanum y $P$. parlatorei presentaron una alta proporción de individuos mayores de $30 \mathrm{~cm}$ de DAP.
Entre los renovales menores se identificaron grupos de especies característicos de cada uno de los extremos del gradiente de complejidad y otros que ocurrieron en la mayor parte de los sitios. Las especies que regeneraron preferentemente en los bosques más simples fueron las mismas que caracterizaron la estructura del dosel en esos sitios (A. acuminata, P. parlatorei y C. tucumanum) (Cuadro 2). Estas especies presentaron densidades muy bajas ( 8 a $20 \mathrm{ind} / \mathrm{ha}$ ). En los grupos que ocurrieron en la mayor parte de las situaciones, aparecieron especies propias del estrato arbóreo inferior ( $S$. peruviana, $S$. grossum, D. lorentzii, Eupatorium lasiophthalmum). El mismo tipo de distribución presentó un grupo con especies propias de los bosques más diversos como $B$. salicifolius, $M$. mato y $P$. tucumanensis. La densidad de las especies de estos grupos varió aproximadamente entre 100 ind/ha y $800 \mathrm{ind} /$ ha y alcanzó valores mayores a
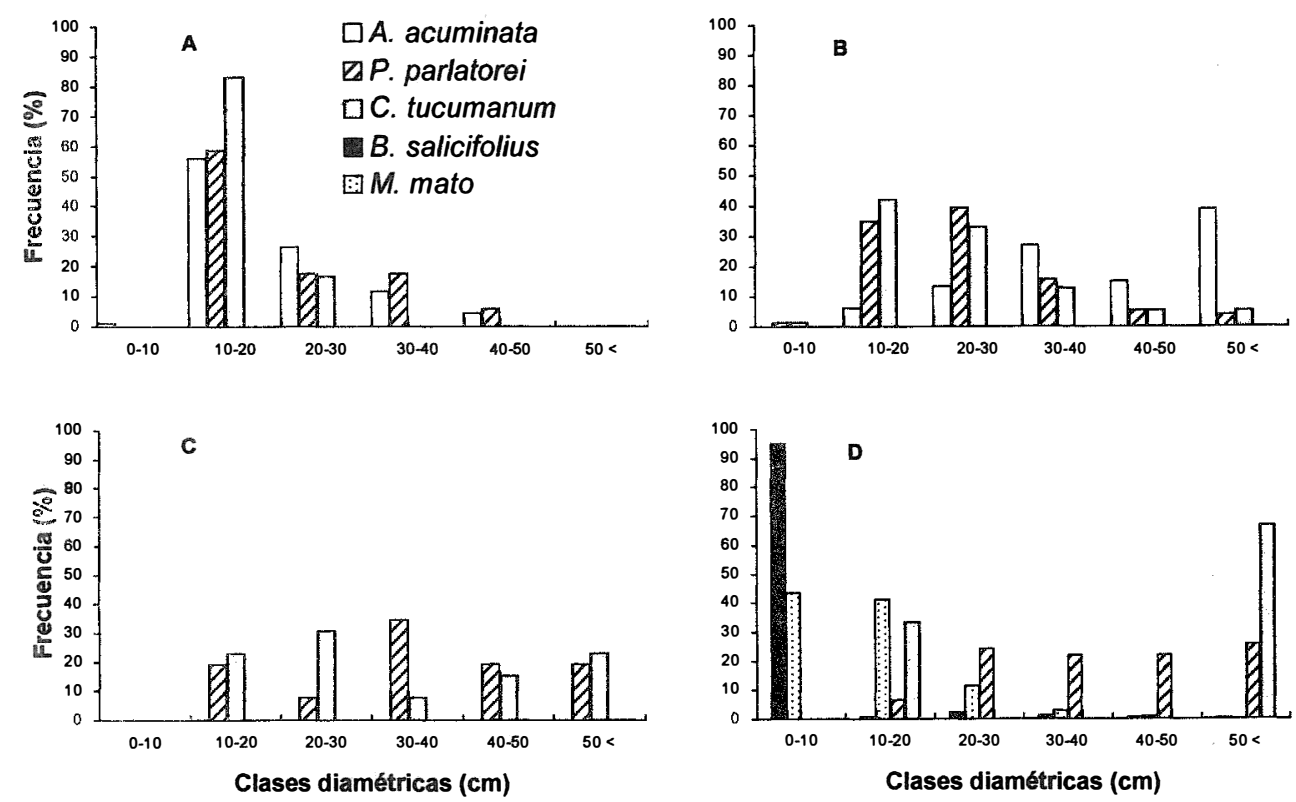

Fig. 2. Distribución por clases diamétricas de las especies más importantes de cada grupo de sitios (A, B, C y D). Los porcentajes se calcularon en base a todos los sitios incluidos en cada grupo.

Diameter distribution for main species of each classification group (A, B, C and D). Percentages were calculated over all sites in each group. 
$2000 \mathrm{ind} / \mathrm{ha}$ en el caso de $S$. grossum. En los bosques más complejos se observaron altas densidades de renovales (100 ind/ha à más de 1 $000 \mathrm{ind} / \mathrm{ha}$ ). El grupo de especies que regeneró en esos sitios estuvo integrado por algunas de sus especies exclusivas (M. pseudomato, A. edulis).

Los grupos de especies formados sobre la base de la densidad de los renovales mayores mostraron las mismas tendencias que los encontrados para los renovales menores. En los sitios menos complejos regeneró principalmente la especie de sotobosque $D$. lorentzii con densidades que variaron entre $50 \mathrm{ind} / \mathrm{ha}$ y $100 \mathrm{ind} / \mathrm{ha}$. En todas las situaciones se registraron otras especies características del estrato inferior como S. peruviana, S. grossum y E. lasiophthalmum alcanzando densidades de $250 \mathrm{ind} / \mathrm{ha}$. En las situaciones intermedias y las más complejas se registraron renovales de las especies propias de los bosques más diversos como J. australis, $C$. lilloi, B. salicifolius y M. mato.

\section{DISCUSIÓN}

Distintos elementos apoyan la hipótesis de que el mosaico de fisonomías boscosas estudiadas responde en alguna medida a un gradiente sucesional. Los bosques se ordenan en el análisis de gradiente siguiendo una tendencia hacia mayor complejidad y diversidad. En ese mismo sentido, algunas especies pioneras o intolerantes según sus distribuciones de tamaños, tienden a ser reemplazadas por especies más tolerantes. Entre las primeras, $A$. acuminata es el caso más claro. Esta especie está ausente en los bosques más diversos y es reconocida por su capacidad de colonizar sitios recientemente perturbados (Grau 1985). P. parlatorei también presenta características de especie pionera ya que su regeneración está asociada a grandes disturbios. Ramadori (en prep.) halló, en otro sector de los Bosques Montanos, que P. parlatore $i$ es una especie que coloniza tempranamente campos de pastoreo abandonados. Esta especie, a diferencia de $A$. acuminata, persiste en los bosques más complejos y diversos. Esto podría deberse a una mayor longevidad de $P$. parlatorei o a una mayor tolerancia a la sombra que le permitiría regenerar en claros grandes dentro del bosque maduro. C. tucumanum parece tener una estrategia similar a $P$. parlatorei, y su persistencia podría estar favorecida por la capacidad de reproducción agámica de esta especie.

En el otro extremo del gradiente, especies como M. mato, Myrcianthes pseudomato, B. salicifolius, $P$. tucumanum, I. argentina, o C.lilloi sólo se encuentran en bosques sin grandes disturbios en las últimas décadas. Estos bosques complejos presentan especies que se encuentran en los bosques de pisos altitudinales más bajos como C. lilloi, B. salicifolius, A. edulis o Myrsine laetevirens (Morales et al. 1995). En consecuencia el gradiente composicional encontrado en parches de los Bosques Montanos resulta similar al gradiente altitudinal Selvas MontanasBosques Montanos (Morales et al. 1995, Grau y Brown 1995).

A pesar de las limitaciones que involucra un estudio basado en cronosecuencias (Pickett 1989), es claro que algunas de las fisonomías boscosas de este nivel altitudinal no presentan una composición estable. En particular los bosques simples de $A$. acuminata y $P$. parlatorei, considerados como característicos de este nivel altitudinal (Cabrera 1976), parecen originarse en disturbios extensos. Estos bosques jóvenes y de baja diversidad serían paulatinamente colonizados por especies más tolerantes dando lugar a la diversidad de fisonomías observadas en el área. De esta manera, la diversidad a escala de paisaje que caracteriza estos bosques (Cabrera 1976, Moyano y Movia 1989), podría estar relacionada con un régimen de disturbios intensos como fuego y pastoreo.

Las evidencias existentes de cambios de pastizal a bosque (Grau 1985, Molinillo y VidesAlmonacid, 1989), y las tendencias de reemplazo de especies encontradas en este trabajo, ponen de manifiesto el carácter dinámico de las fisonomías de los Bosques Montanos. El manejo de este tipo de ambientes requiere del conocimiento de los patrones temporales y espaciales de los cambios en las comunidades (Turner $e t$ al. 1994, Baker 1992, Romme 1982). Existe 
muy poca información sobre los mecanismos de regeneración post-disturbio de las especies más importantes, los mecanismos de reemplazo de especies durante la sucesión y los factores climáticos y antrópicos que inciden sobre el régimen de disturbios. Las estrategias de conservación deben contemplar este tipo de información ya que distintos tipos de manejo podrían modificar los regímenes de disturbio y afectar la diversidad beta del área.

\section{AGRADECIMIENTOS}

Este trabajo contó con la financiación parcial de la Universidad Nacional de Tucumán y el Consejo Nacional de Investigaciones Científicas y Tecnológicas (CONICET, Argentina). El trabajo de campo fue apoyado por el personal de Protección, y de Obras y Servicios del Parque Biológico Sierra de San Javier, Universidad Nacional de Tucumán, Argentina.

\section{RESUMEN}

Se estudió la composición y estructura de tamaños de las especies arbóreas en diferentes fisonomías boscosas de los "Bosques Montanos" de la Sierra de San Javier, en el subtrópico de Argentina entre 1600 y 1800 msnm. En un análisis de correspondencia, los sitios se ordenaron siguiendo un gradiente de riqueza y complejidad. Las especies características de los sitios más diversos como las mirtáceas mostraron distribuciones diamétricas en "J-invertida", lo que sugiere regeneración continua. Por el contrario, las especies características de los bosques simples como $\mathrm{A}$. acuminata, $P$. parlatorei y $C$. tucumanum, presesentaron distribuciones diamétricas unimodales con baja proporción de individuos menores. La densidad de individuos menores de $10 \mathrm{~cm}$ de DAP muestran que $A$. acuminata, $P$. parlatorei y $C$. tucumanum constituyen especies pioneras que colonizarían áreas no arboladas originando bosques simples. Estas especies son progresivamente reemplazadas por especies tolerantes durante la sucesión originando diversas fisonomías. La diversidad a escala de paisaje de los Bosques Montanos se originaría en eventos discretos de reclutamiento de las especies pioneras, seguidos de sucesión hacia bosques dominados por especies tolerantes.

\section{REFERENCIAS}

Baker, W.L. 1992. The landscape ecology of large disturbances in the design and management of nature reserves. Landscape Ecol. 3: 181-194

Brown, A.D. 1995. Fitogeografía y conservación de las selvas de montaña del noroeste de Argentina, p. 663-672. In S.P. Churchill, H. Balslev, E. Forero \& J. Luteyn (eds.). Biodiversity and Conservation of Neotropical Montane Forests. New York Botanical Garden, Bronx.

Cabrera, A.L. 1976. Regiones Fitogeográficas Argentinas. ACME, Buenos Aires, Argentina. 85 p.

Grau, A. 1985. La expansión del aliso del cerro (Alnus acuminata HBK subsp acuminata) en el Noroeste de Argentina. Lilloa 36: 237-247.

Grau, H.R. 1989. El distrito de los bosques montanos y su importancia para manejo y conservación en la provincia de Tucumán. Serie Monográfica y Didáctica FCN-UNT 1: $1-10$.

Grau, H.R. \& A.D. Brown. 1995. Patterns of tree species diversity along latitudinal and altitudinal gradients in the Argentinean subtropical montane forests, p. 295300. In S.P. Churchill, H. Balslev, E. Forero \& J.L. Luteyn (eds.). Biodiversity and Conservacion of Netoropical Montane Forests. New York Botanical Garden, Bronx, USA.

Hill, M.O. 1979. TWINSPAN - A FORTRAN program for arranging multivariate data in an ordered two way table by classification of individuals and attributes. Cornell University Ithaca New York, $90 \mathrm{pp}$.

Holdridge, L.R. 1978. Ecología Basada en Zonas de Vida IICA, San José, Costa Rica. 216 p.

Hueck, K. 1978. Los Bosques de Sudamérica. Ecología, Composición e Importancia Económica. Agencia Alemana de Cooperación Técnica (GTZ). Berlín, 451 p.

Molinillo, M.F. y R. Vides-Almonacid. 1989. Uso de los recusos naturales en el bosque de Aliso de la provincia de Tucumán. Avances de Investigación, INGEMA 1: 1 20.

Morales, J.M.; M. Sirombra \& A.D. Brown. 1995. Riqueza de árboles en las Yungas Argentinas, p. 163-174. In A.D. Brown y H.R. Grau. (eds.). Investigación, Conservación y Desarrollo en Selvas Subtropicales de Montaña. LIEY, Tucumán, Argentina. 
Moyano, M.Y. \& C.P. Movia. 1989. Relevamiento fisonómico-estructural de las sierras de San Javier y El Periquillo (Tucumán-Argentina), I. Area de las Yungas. Lilloa 37: 123-135.

Pickett, S.T.A. 1989. Space-for-time substitution as an alternative to long-term studies, p. 110-135. In G.E. Likens (ed.). Long-term Studies in Ecology. Springer-Verlag Nueva York.

Pickett, S.T.A. \& P.S. White. 1985. The Ecology of Natural Disturbances and Patch Dynamics. Academic, Orlando, Florida.

Romme, W.H. 1982. Fire and landscape diversity in subalpine forests of Yellowstone National Park. Ecol. Monog. 52: 199-221.
Swaine, M.D. \& T.C. Whitmore, 1988. On the definition of ecological species groups in tropical rain forest Vegetatio 75: 81.86.

ter Braak, C.J.F. 1987. Ordination, p. 91-133. In Jongman, R.H.G., C.J.F. ter Braak y O.F.R. van Tongeren (eds.). Data analysis in community and landscape ecology. Cambridge University, Cambridge.

Turner, M.G.; W.H. Romme \& R.H. Gardner. 1994. Landscape disturbance models and the long-term dynamics of natural areas. Natur. Areas J. 14: 3-11

Veblen, T.T. 1992. Regeneration dynamics, p. 152-187. In D.C. Glenn-Lewin, R.K. Peet \& T.T. Veblen (eds.). Plant Succession, Theory and Prediction. Chapman \& Hall, Nueva York. 